\title{
Commentary on "Methenamine hippurate compared with trimethoprim for the prevention of recurrent urinary tract infections: a randomized clinical trial"
}

\author{
Abdelmageed Abdelrahman ${ }^{1}$ \\ Received: 26 May 2021 / Accepted: 12 June 2021 / Published online: 6 July 2021 \\ (C) The International Urogynecological Association 2021
}

This was a non-blinded randomized trial comparing methenamine hippurate with trimethoprim for the prevention of recurrent urinary tract infections 12 months after starting treatment [1]. Inclusion criteria were women over 18 years who had had at least two culture-positive urinary tract infections in the prior 6 months or three in the past year. Patients were excluded if pregnant or if they had had any urinary tract abnormalities, acute pyelonephritis, or renal insufficiency or failure. Other exclusion criteria include allergy to medication or if on prophylactic antibiotics for post-coital recurrent urinary tract infection. The primary outcome of this study was culture-proven urinary tract infection recurrence by 12 months after initiating prophylaxis.

There was a total of 86 patients who were included in the final analysis. Results of the study showed that there was no difference between groups with regard to recurrent urinary tract infections, with $65 \%$ recurrence in the trimethoprim group versus $65 \%$ recurrence in the methenamine hippurate group. The authors concluded that methenamine hippurate

Abdelmageed Abdelrahman

abdelmageed@hotmail.co.uk

1 Department of Urogynaecology, Liverpool Women's Hospital NHS Foundation Trust, Liverpool, UK may be an alternative for the prevention of recurrent urinary tract infections, with similar rates of recurrence and adverse effects to trimethoprim.

The results of the study are promising in that methenamine hippurate may be considered as a first-line therapy, does not have the potential to cause the same antibiotic resistance as low-dose antibiotics, and has efficacy in preventing recurrent urinary tract infections. Strengths of the study include the randomized trial design with generalizable results, as the inclusion criteria represented a commonly seen population. Limitations of the study include the variability in follow-up and that the study was not blinded.

\section{Declarations}

Conflicts of interest None.

\section{Reference}

1. Botros C, Lozo S, Iyer S, et al. Methenamine hippurate compared with trimethoprim for the prevention of recurrent urinary tract infections: a randomized clinical trial. Int Urogynecol J. 2021. https://ink. springer.com/article/10.1007/s00192-021-04849-0

Publisher's note Springer Nature remains neutral with regard to jurisdictional claims in published maps and institutional affiliations. 